\title{
EFECTO DE LAS PRÁCTICAS AGRONÓMICAS EN LAS PROPIEDADES DEL SUELO EN LADERAS 1
}

\author{
Claro A. Alfonso ${ }^{2}$, Mario Riverol ${ }^{2}$, Pedro Porras ${ }^{3}, E_{\text {Eduardo Cabrera }}^{3}$, Víctor Somoza ${ }^{2}$, Alberto Otero ${ }^{3}$
}

\section{RESUMEN}

Efecto de las prácticas agronómicas en las propiedades del suelo en laderas. En un suelo Ultisol de textura arenosa, fuertemente erosionado, de baja fertilidad en fósforo, materia orgánica y ácido, ubicado en la localidad de La Leña en la provincia de Pinar del Río, en la República de Cuba, se realizó durante el año 1996 un ensayo en dos segmentos de microcuencas, en las que se implementaron medidas de conservación de suelos consistentes en barreras vivas de Gliricidia sepium L. (primer segmento) y Vetiveria zizanoide L. (segundo segmento) complementadas con coberturas de maíz asociado con Mucuna d. y Canavalia e., como precedentes del frijol. Los resultados mostraron ligeros cambios de la pendiente intrabarreras con respecto al valor inicial (10 y 9\%). Los valores de la biomasa seca total de las asociaciones fueron de 17 y $15 \mathrm{t} / \mathrm{ha}$, la velocidad de cubrimiento a los 30 días fue mayor al $70 \%$, la colonización radical profusa presentada por las asociaciones indican una mejora de la red poral del suelo, reflejándose en la tendencia positiva del estado interno de la estructura que evoluciona hacia un estado $(\mathrm{G})$ poroso. Los rendimientos de maíz fueron de 2900 y $2500 \mathrm{~kg} / \mathrm{ha}$ y los de frijol de 1800 y $1630 \mathrm{~kg} / \mathrm{ha}$. Valores considerados elevados, si se tiene en cuenta el grado de deterioro de este suelo.

\begin{abstract}
Effect of agronomic practices in soil properties in hillsides . Trials were established in a highly eroded Ultisol soil of sandy loam texture, of poor fertility, acid, and low in phosphorus and organic matter, located in the locality of La Leña in Pinar del Río province, Republic of Cuba. A study was carried ont in 1996 in two watersheds, in which were implemented conservation practices consisting of live barriers of Gliricidia Sepium L. (in the first watershed) and Vetiveria Ziznoide $\mathrm{L}$. (in the second watershed) complemented with ground cover of maize associated with legumes followed by beans in succession. Results showed slight changes in slope between barriers with respect to the initial value (10\% vs $9 \%$ ). Values of total dry biomass in the associations were 17 and $15 \mathrm{t} / \mathrm{ha}$, and ground coverage at 30 days was more than $70 \%$. Profuse root colonization presented by the associations indicated an improvement in porosity of the soil, reflecting a positive tendency in the internal structure that has evolved to a porous state. Yields of maize were 2900 and $2000 \mathrm{~kg} / \mathrm{ha}$, and of bean were 1800 and $1630 \mathrm{~kg} / \mathrm{ha}$, which are relatively high values, considering the degraded condition of the soil.
\end{abstract}

\section{INTRODUCCIÓN}

Para lograr hacer sostenible cualquier sistema de producción es necesario tener en cuenta la estrecha interrelación multidireccional que se establece entre sus componentes. La modificación de uno de ellos puede afectar los otros (CIAT. 1994, citado por Henríquez et al 1992), en consecuencia, el rendimiento, se verá reducido. Por esta razón se recomienda el manejo integrado de todas las partes constitutivas del sistema de cultivo (Agricultura Sostenible), según Altieri (1995).
Un componente de tal sistema que tiene relación directa y limita la producción, por sí sola, es la erosión en condiciones de laderas, fenómeno que al empobrecer los suelos por deterioro químico, físico, pérdidas de materia orgánica, etc. , repercute en la obtención de bajos rendimientos de los cultivos. De acuerdo con lo anterior, se vislumbra la factibilidad técnica para aumentar la producción de los cultivos de frijol y maíz de esa provincia, en beneficio de un gran número de productores que explotan los suelos en laderas, de forma intensa. Este aumento tendrá un impacto sobre la super-

\footnotetext{
1 Presentado en la XLV Reunión Anual del PCCMCA, Guatemala 1999.

2 Estación Experimental La Renee del Instituto de suelos del MINAG. Km $33^{1 / 2}$ carretera de Bejucal a Quivicán. Habana Cuba. E-mail: larenee@ceniai.inf.cu

3 Estación Experimental de San Juan y Martínez, Finca Obeso, Pinar del Río. Cuba.
} 
ficie sembrada, ya que estimulará a los agricultores a utilizar terrenos ociosos, que presentan una fuerte degradación del suelo asociada a la erosión hídrica.

\section{MATERIALES Y MÉTODOS}

Medición, cuantificación y establecimiento de las tasas de erosión producidas por las diferentes prácticas agronómicas establecidas de febrero de 1996 a enero de 1997.

La localidad seleccionada para ejecutar el ensayo, se encuentra en la Comunidad La Leña. En el Cuadro 1 se describen las características de las localidades prácticas agronómicas realizadas así como la rotacion de cultivos.

Los segmentos de microcuencas de La Comunidad La Leña no poseen equipos para la determinación de las pérdidas. Se determinó la variación de la pendiente promedio mediante levantamiento altimétrico, y se midió el Espesor Máximo de Sedimentos (EMS). Se hizo mayor énfasis en la evaluación del deterioro de la fertilidad y en la validación de las mejores combinaciones de medidas que ya venían estudiándose en esta Comunidad. El diseño experimental que se utilizó en cada segmento de microcuenca para evaluar el rendimiento, fue el de bloques al azar, con cuatro parcelas de seis surcos por diez metros de largo, repartidas al azar en las terrazas de cada segmento. Estas constituyeron estaciones fijas, referenciadas en el terreno, para el seguimiento de todas las observaciones y mediciones que se efectuaron.

Las observaciones que se realizaron para determinar el deterioro fueron:
Para el suelo: Estado interno de la estructura, método cartográfico de Manichon (1982a), modificada por Alfonso (1982), porosidad textural y estructural, según técnica de Monnier et al (1973), permeabilidad con infiltrómetro de laboratorio, variación de: la acidez, los macronutrimentos, la materia orgánica.

Todas estas observaciones se efectuaron anualmente, y replicadas tres veces.

Para la planta: Colonización radical de las asociaciones, según Método Cartográfico de Tardieu (1985), modificado por Alfonso (1987), dinámica de cubrimiento de la composición botánica, según Da Veiga y Do Prado (1993), biomasa aérea y subterránea producida por las asociaciones, extracción reintegrable de nutrimentos.

El sistema de las terrazas de barreras vivas se estableció con un intervalo vertical de un metro, lo cual en las pendientes seleccionadas de 9 - $10 \%$, arrojó una distancia aproximada de 15 a $20 \mathrm{~m}$. entre bordos. Bajo este sistema, parte de los residuos, se colocarán en el camellón sobre la base de las barreras, el resto en la preparación. Los ensayos establecidos en segmentos de microcuencas se manejaron con el cultivo del maíz en primavera en relevo con el frijol común. Con este patrón tradicional de cultivos, se evaluó el sistema de terraza de barreras vivas complementadas con las asociaciones del maíz con Canavalia e. y Stizolobium d. El marco de siembra del maíz (variedad T - 66) fue de 35 cm entre plantas x $90 \mathrm{~cm}$ entre líneas de siembra y las leguminosas se sembraron a los 12 - 15 días de ésta, en las calles, inmediatamente después del primer aporque, su marco de siembra fue a $15-20 \mathrm{~cm}$ entre plantas en todas las calles. La fecha de siembra fue para los dos segmentos en la primera quincena de mayo.

Cuadro 1. Características del ensayo y tratamientos ejecutados. Cuba. 1996-1997.

\begin{tabular}{|c|c|c|c|c|c|c|}
\hline Estación Experimental & Localidad & MSNM & \multicolumn{2}{|c|}{ Tipo de suelos } & \multicolumn{2}{|c|}{ Tipo de instalación } \\
\hline Consolación del Sur & Comunidad & $50-60$ & \multicolumn{2}{|c|}{$\begin{array}{l}\text { Ferralítico Cuarcítico Amarillo } \\
\text { Lixiviado (Ultisol) }\end{array}$} & \multicolumn{2}{|c|}{$\begin{array}{l}\text { Segmentos de microcuencas sin } \\
\text { instalación, dos segmentos. }\end{array}$} \\
\hline Tratamientos & febrero-marzo & abril-mayo & junio-julio & agosto-set. & octubre & nov.-dic.-ene \\
\hline S/Mc 1 (Gliricidia s.) & Barbecho & Prep. Suelos & Maíz-Stizozol d. & Barbecho & Preparación suelos & Frijol \\
\hline S/Mc 2 (Vetiveria $\mathrm{z})$. & Barbecho & Prep. Suelos & Maíz+Canavalia e. & Barbecho & Preparación suelos & Frijol \\
\hline
\end{tabular}

Prácticas agronómicas realizadas:

\begin{tabular}{lccclc}
\hline Localidad & Tratamientos & Pend. (\%) & Area & \multicolumn{1}{c}{ Práctica } & Rotación \\
\hline La Leña & S/Mc - 1 & 10 & 1 ha & $\begin{array}{l}\text { Bordos protegidos con barreras } \\
\text { vivas de Gliricidia sepium }\end{array}$ & Maíz + Stizolobium - Frijol \\
& S/Mc - 2 & 9 & 0,9 ha & $\begin{array}{l}\text { Bordos protegidos con barreras } \\
\text { vivas de Vetiveria zizanoide }\end{array}$ & Maíz + Canavalia e. - Frijol \\
\hline
\end{tabular}


El frijol se sembró a mediados de noviembre para el segmento uno y a mediados de diciembre para el segmento dos, el marco de siembra fue de $70 \mathrm{~cm}$ x $5 \mathrm{~cm}$, la variedad utilizada: Tomeguín 93.

La fertilización se realizó según lo recomendado por el Instructivo Técnico para el cultivo del frijol y del maíz, aportándose todo el $\mathrm{P}$ y $\mathrm{K}$ de fondo en la siembra, y el $\mathrm{N}$ fraccionado al inicio y a los 30 días de la primera fertilización.

Cada sitio se caracterizó mediante la medición de la pendiente del terreno y la descripción de un perfil del suelo, donde se tomaron muestras de los horizontes para análisis de rutina.

\section{RESULTADOS Y DISCUSIÓN}

\section{Modificaciones de la pendiente y espesor máximo de los sedimentos (EMS)}

Las pérdidas se definieron al evaluar la variación de la pendiente media intrabarreras a la determinación del espesor máximo de los sedimentos (EMS) en la sección receptora de las barreras. Los resultados de estas mediciones se presentan en el Cuadro 2, donde se observa que en el segmento uno, con barreras vivas de Gliricidia sepium establecidas a inicios de 1994, al cabo de tres años de desarrollo, tienen una disminución en la pendiente media intrabarrera que va de $10 \%$ a 9,3\% y el espesor máximo de sedimentos (EMS) adquiere el valor de $35 \mathrm{~cm}$. Estas cifras contrastan con las obtenidas por Francisco (1995), quien encontró (EMS) de $27 \mathrm{~cm}$ en un suelo Mollisol de textura arcillosa de la región de Los Tuxtlas, en México, diferencias que pueden explicarse debido a las texturas opuestas de ambos suelos (el de La Leña es loam arenosos), la que les confiere una baja estabilidad estructural, por lo que la energía cinética de las lluvias las hace más dispersante y erosivas. Por otro lado, el manejo que se realiza con el establecimiento de las barreras, al crear bordos de desagüe sobre los que se siembran las mismas para su protección. Esta práctica ayuda desde el mismo co- mienzo a la detención de las escorrentías, suavizando la velocidad de escurrimiento.

En el caso del segmento dos, con barreras vivas de Vetiveria zizanoide, L. establecidas a finales de 1994, no mostró ninguna variación en la pendiente media, después de dos años, teniendo un (EMS) de $10 \mathrm{~cm}$. Estos resultados se explican por el manejo de estas barreras, en las mismas no se trazaron bordos de desagüe para su montaje, por lo que su desarrollo ha sido muy lento. Se añade a esta causa el poco tiempo que llevan de establecidas.

Estos resultados indican que los bordos protegidos con barreras vivas, efectúan una buena intersección de los sedimentos movilizados por las escorrentías, siendo más significativas en las de Gliricidia $s$. que en las de Vetiveria $z$., por los motivos antes enunciados.

\section{Comportamiento de las asociaciones de maíz y legu- minosas.}

Como complemento de las barreras vivas, se utilizaron como cubiertas vegetales, las asociaciones de maíz con Stizolobium deeringianum L, para el segmento uno y Canavalia ensiformis L. para el segmento dos respectivamente. La decisión de validar estas dos leguminosas como más promisorias en el ensayo del año 1996, se debe a los resultados obtenidos con las mismas en las investigaciones llevadas a cabo en el trienio, 1993 - 1995, en las localidades de Campo Hermoso, Calderón y La Leña respectivamente (Alfonso et al. 1997), coincidentes con los reportes de Francisco et al (1995) en la región de los Tuxtlas en México y García et al (1997) para Cuba. El comportamiento de estas coberturas (Cuadro 3), muestra que la velocidad de cubrimiento de ambas leguminosas alcanza más del 70\% a partir de los 30 días de germinados, produciendo una reducción casi total al cabo de los 50 días de las malezas y de los espacios vacíos sin plantas, estos resultados son similares a los obtenidos por Marín (1996) para la Canavalia. La dinámica de crecimiento de la Canavalia fue un poco más lenta, por las características propias de esta especie.

Cuadro 2. Pendientes medias y espesor máximo de sedimentos (EMS) en las terrazas de muros vivos. La Leña, Cuba, febrero de 1997.

\begin{tabular}{|c|c|c|c|c|c|}
\hline \multirow{2}{*}{$\begin{array}{c}\text { Año de establecimiento de las barreras } \\
\text { vivas }\end{array}$} & \multicolumn{2}{|c|}{ Pendiente $(\%)$} & \multirow{2}{*}{$\begin{array}{c}\text { Avance } \\
(\%)\end{array}$} & \multirow{2}{*}{$\begin{array}{l}\text { EMS } \\
(\mathbf{c m})\end{array}$} & \multirow{2}{*}{$\begin{array}{c}\text { Lluvias } \\
(\mathbf{m m})\end{array}$} \\
\hline & Original & Actual & & & \\
\hline Segmento 1 Gliricidia s. (1994) & 10 & 9,3 & 7 & 35 & 1624 \\
\hline Segmento 2 Vetiveria z. (1995) & 9 & 9 & 0 & 10 & 1624 \\
\hline
\end{tabular}


Cuadro 3. Velocidad de cubrimiento de la asociación. Cuba. 1996-1997.

\begin{tabular}{|c|c|c|c|c|c|c|c|c|c|c|c|c|c|c|}
\hline \multirow{2}{*}{$\begin{array}{l}\text { Componente } \\
\text { Segmento } 1\end{array}$} & \multicolumn{2}{|c|}{7 días } & \multicolumn{2}{|c|}{14 días } & \multicolumn{2}{|c|}{21 días } & \multicolumn{2}{|c|}{28 días } & \multicolumn{2}{|c|}{35 días } & \multicolumn{2}{|c|}{42 días } & \multicolumn{2}{|c|}{49 días } \\
\hline & No & $\%$ & No & $\%$ & No & $\%$ & No & $\%$ & No & $\%$ & No & $\%$ & No & $\%$ \\
\hline Stizolobium & 44 & 17 & 89 & 35 & 118 & 46 & 159 & 62 & 197 & 77 & 202 & 79 & 210 & 82 \\
\hline Malezas & 51 & 20 & 49 & 19 & 46 & 18 & 46 & 18 & 36 & 14 & 41 & 16 & 36 & 14 \\
\hline Vacíos & 161 & 63 & 118 & 46 & 92 & 36 & 51 & 20 & 23 & 9 & 13 & 5 & 10 & 4 \\
\hline Segmento 2 & & & & & & & & & & & & & & \\
\hline Canavalia e. & 49 & 19 & 82 & 32 & 115 & 45 & 154 & 60 & 195 & 76 & 200 & 78 & 205 & 80 \\
\hline Malezas & 56 & 22 & 49 & 19 & 44 & 17 & 49 & 19 & 38 & 15 & 41 & 16 & 38 & 15 \\
\hline Vacíos & 153 & 59 & 125 & 49 & 97 & 38 & 54 & 21 & 23 & 9 & 15 & 6 & 13 & 5 \\
\hline
\end{tabular}

No $=$ Número de cuadrículas $\%=$ Por ciento del total de cuadrículas (90)

En consonancia con estos resultados, la producción de biomasa seca total (Figura 1), donde el Stizolobium $d$. tuvo una mayor magnitud que la producida por la Canavalia e.. Sin embargo, los pesos obtenidos por los rastrojos de maíz (cañas o tallos) fueron casi iguales, cuantificadas estas cifras antes del enterramiento de la asociación (90 días de sembrado el maíz).

En cuanto al reciclaje de los nutrimentos que pueden efectuar ambas leguminosas (Cuadro 4), se relaciona de forma estrecha con la producción de la biomasa seca total, por lo que los aportes del Stizolobium son mayores que los de la Canavalia. Las concentraciones porcentuales son muy parecidas para ambas legumino-

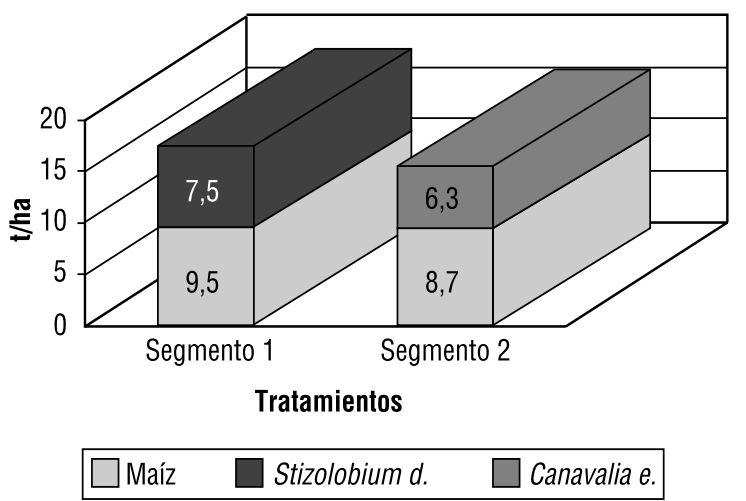

Figura 1. Producción de biomasa seca total. La Leña. 1996. sas. Evidentemente los tenores de N,P,K y Ca en forma orgánica, permitirán sustituir y balancear las fertilizaciones tradicionales que se realizan en los cultivos de maíz y frijol.

\section{Modificaciones de las propiedades del suelo bajo prácticas de conservación}

Desde el punto de vista de la fertilidad general, los valores reportados en el Cuadro 5, permiten aseverar que éste es un suelo de baja fertilidad. Al comparar los datos obtenidos en el año 1996 con los del 1995, se observa un ligero avance en los tenores de materia orgánica, no así en P y K, desde el punto de vista de la fertilidad física, reportando una porosidad global poco diferenciada, pero en su composición se pudo ver un incremento de la macroporosidad o porosidad estructural, en detrimento de la microporosidad o porosidad textural, lo que facilita la colonización de las raíces y la circulación del agua con los nutrimentos y el aire a su través. Esta característica le confiere y así lo expresan los valores, un ligero incremento de la permeabilidad y una disminución de la compactación.

Completan esta apreciación las observaciones realizadas al estado interno de la estructura, mediante perfiles cartográficos (Cuadro 6), siguiendo la metodología propuesta por Manichon (1982a), modificada por Alfonso et al. (1987), donde se notó un ligero

Cuadro 4. Reciclaje de nutrimento efectuados por las asociaciones y las malezas, Cuba. (1996).

\begin{tabular}{cccccrr}
\hline Segmentos & Tratamientos & Concentración & N & P & K & Ca \\
\hline \multirow{2}{*}{ Mc. 1 } & Stizolobium d. & $\%$ & 2,5 & 0,35 & 2,4 & 1,46 \\
& & $\mathrm{~kg} . / \mathrm{ha}$ & 160 & 22.4 & 154 & 93 \\
Mc. 2 & Canavalia $\mathrm{e}$ & $\%$ & 2,8 & 0,42 & 2,5 & 1,43 \\
& & $\mathrm{~kg} . / \mathrm{ha}$ & 115 & 17 & 103 & 59 \\
\hline
\end{tabular}


Cuadro 5. Modificaciones de algunas propiedades del suelo. La Leña, Cuba. 1996.

\begin{tabular}{|c|c|c|c|c|c|c|c|c|c|c|c|c|c|}
\hline \multirow[t]{2}{*}{ Tratamientos } & \multirow[t]{2}{*}{ Suelo } & \multirow[t]{2}{*}{ Textura } & \multirow{2}{*}{$\begin{array}{r}\text { Prof. } \\
(\mathbf{c m})\end{array}$} & \multirow[t]{2}{*}{$\mathbf{p H}$} & \multirow{2}{*}{$\begin{array}{l}\text { M.O. } \\
(\%)\end{array}$} & \multirow{2}{*}{$\begin{array}{c}\text { P } \\
\text { ppm }\end{array}$} & \multirow{2}{*}{$\begin{array}{c}\text { K } \\
\text { ppm }\end{array}$} & \multicolumn{3}{|c|}{ Porosidad (\%) } & \multicolumn{2}{|c|}{ Permeabilidad } & \multirow{2}{*}{$\begin{array}{c}\text { Compact } \\
\text { Kg./cm² } \\
\text { Surco }\end{array}$} \\
\hline & & & & & & & & PG & PT & $\mathbf{P E}$ & $\begin{array}{l}\mathbf{m m} / \mathbf{h} \\
\text { Surco }\end{array}$ & $\begin{array}{c}\mathrm{mm} / \mathrm{h} \\
\text { Calle }\end{array}$ & \\
\hline $\begin{array}{l}\text { Segmento Mc } 1 \\
\text { Barreras vivas de }\end{array}$ & $\begin{array}{c}\text { Ferr. Cuarc. } \\
\text { Amarillo }\end{array}$ & $\begin{array}{r}\text { Loam } \\
\text { Arenosa }\end{array}$ & $0-10$ & 4,7 & 1,00 & 8,6 & 2,1 & 48 & 38 & 10 & & & \\
\hline \multirow[t]{3}{*}{ Gliricidia s. } & (Ultisol) & & $11-20$ & 4,6 & 0,80 & 4,3 & 8 & 46 & 37 & 9 & 125 & 85 & 3,00 \\
\hline & & & $21-30$ & 4,4 & 0,50 & 2 & 2 & 32 & 29 & 3 & & & \\
\hline & & & $31-40$ & 4,5 & 0,20 & 0,5 & 2 & 30 & 28 & 2 & & & \\
\hline Segmento Mc 2 & Ferr. Cuarc. & Loam & $0-10$ & 4,5 & 1,00 & 9 & 3 & 53 & 43 & 10 & & & \\
\hline \multirow[t]{3}{*}{ Sin barreras viva } & Amarillo & Arenosa & $11-20$ & 4,4 & 0,70 & 5 & 5 & 48 & 40 & 8 & 95 & 60 & 3,25 \\
\hline & (Ultisol) & & $21-30$ & 4,5 & 0,30 & 3 & 2 & 42 & 40 & 2 & & & \\
\hline & & & $31-40$ & 4,6 & 0,20 & 1 & 32 & 30 & 2 & & & & \\
\hline
\end{tabular}

$\mathrm{PG}=$ Porosidad Global $\quad \mathrm{PT}=$ Porosidad Textural $\quad \mathrm{PE}=$ Porosidad Estructural $\quad(\mathrm{PG}-\mathrm{PT}=\mathrm{PE})$

Permeabilidad se realizó con infiltrómetro de laboratorio y la compactación con Penetrómetro de bolsillo.

Cuadro 6. Modificaciones del estado interno de la estructura Microcuenca La Leña, Cuba (1996).

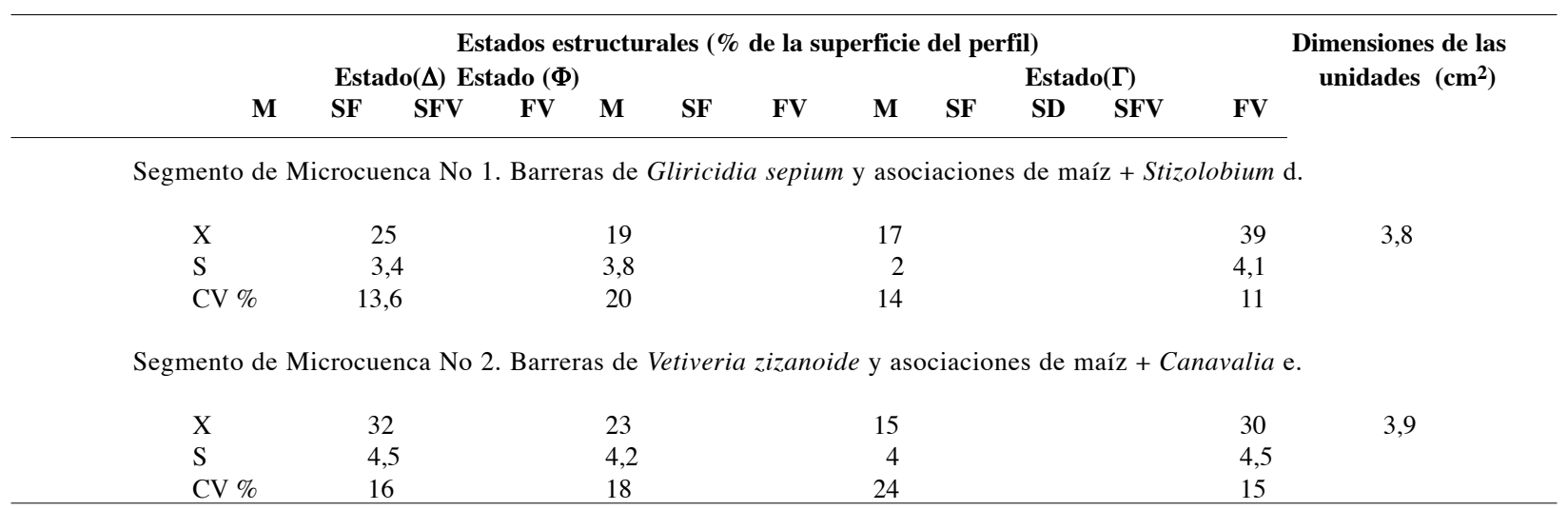

Valores promedios de tres perfiles

Definiciones indicativas de los estados internos de la estructura

Estado $(\Delta)=$ Aspecto continuo compacto.

Estado $(\Phi)=$ Presencia de fisuras en una unidad masiva $(\Delta)$

Estado $(\Gamma)=$ Agregados visibles de gran porosidad

Cuando muchos elementos estructurales iguales de dimensiones variables constituyen zonas

pueden presentarse:

$\mathrm{M}=$ Masivo, aspecto de una sola unidad formando la zona.

$\mathrm{SD}=$ Elementos estructurales de difícil apreciación

$\mathrm{SF}=$ Elementos estructurales soldados de fácil apreciación.

$\mathrm{F}=$ Estructura fragmentaria compuesta por elementos individuales.

$\mathrm{V}=$ Grandes vacíos entre los elementos estructurales o dentro de estos.

incremento de zonas del tipo $(\mathrm{G})$ porosas, en el segmento uno con respecto a las vistas en el segmento dos, en relación con las zonas (D) masivas de estructura más compacta, son mayores en este último segmento, no obstante aunque con pequeños matices en ambas áreas se observa una mejora de la fertilidad general y del estado físico en particular. Este comportamiento puede deberse a que las condiciones físicas de estos suelos loam arenosos, debido a la presencia de materia orgánica que se reporta, crean pseudoagregados relati- vamente más estables que las partículas arenosas simples, poco o nada coherentes por sí mismas. Estas reestructuraciones tiene consecuencias directas sobre la estabilidad estructural de estos suelos, haciéndolos menos vulnerables a la erosión, según reportan Alfonso (1987), Collinet y Mazariego (1996). Por su parte Turrents et al. (1995); Uribe y Francisco (1993), en suelos Mollisoles y Hapludalf, de la región de los Tuxtlas, en similares circunstancias de manejo, reportan variaciones favorables de las propiedades del suelo. 
Por último como se dijo anteriormente, la colonización radical (Cuadro 7), se vio favorecida al mejorar el estado físico o la fertilidad física en particular, como se dijo antes, al verse una mayor colonización en el segmento uno sometido a prácticas mejoradoras desde hace más tiempo, notándose raíces de leguminosas al nivel de los $30 \mathrm{~cm}$, mientras que las del maíz no van más allá de los $20 \mathrm{~cm}$. Esto responde, a las modificaciones del estado interno de la estructura, en el segmento uno fue menos impactante. Estos resultados, al ser comparados con los obtenidos por Alfonso et al (1997), en áreas aledañas dedicadas al cultivo del tabaco, desde hace muchos años, muestran la recuperación lenta, pero perceptible del estado estructural en general y de un restablecimiento de la fertilidad natural, que no se observa en suelos sin prácticas de conservación. La respuesta de este suelo a las prácticas de mejoramiento conservacionista, hacen pensar en la posibilidad real de establecer un sistema de producción sostenible para la producción del frijol en rotación con el maíz en relevo,

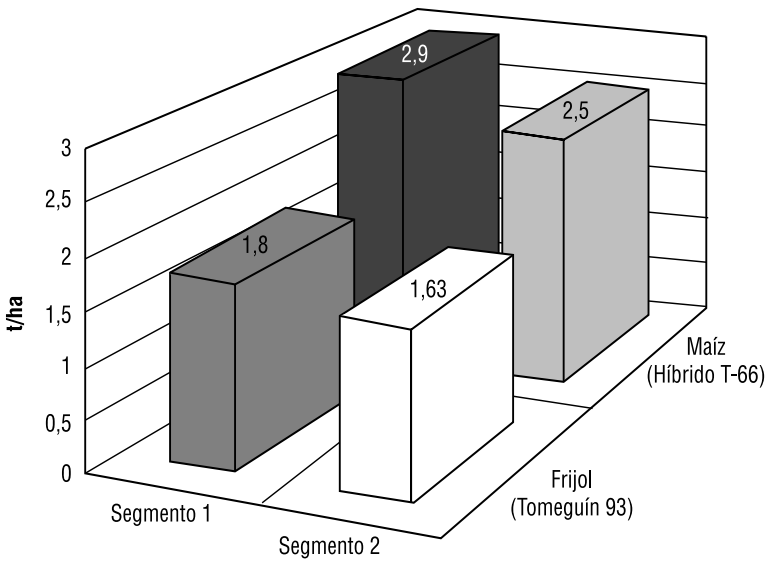

Figura 2. Rendimientos en granos de maíz yfrijol. La Leña. 1996. en las condiciones de la Comunidad La Leña.

Cuadro 7. Colonización radical de los cultivos intercosechas de La Leña. 1996.

\begin{tabular}{|c|c|c|c|c|c|c|c|c|}
\hline \multirow{3}{*}{$\begin{array}{c}\text { Profundidad } \\
\text { Capas }(\mathbf{c m})\end{array}$} & \multicolumn{4}{|c|}{ Segmento de Microcuenca No 1.} & \multicolumn{4}{|c|}{ Segmento de Microcuenca No 2 . } \\
\hline & \multicolumn{2}{|c|}{ Maíz } & \multicolumn{2}{|c|}{ Stizolobium d. } & \multicolumn{2}{|c|}{ Maíz } & \multicolumn{2}{|c|}{ Canavalia e. } \\
\hline & No C. & $\%$ & No C. & $\%$ & No C. & $\%$ & No C. & $\%$ \\
\hline $0-10$ & 135 & 74,6 & 59 & 31,2 & 126 & 79,8 & 48 & 31,4 \\
\hline $11-20$ & 42 & 23,08 & 85 & 44,9 & 32 & 20 & 78 & 51,0 \\
\hline $21-30$ & 5 & 2,75 & 31 & 16,4 & & & 19 & 12,4 \\
\hline $31-40$ & & & 14 & 7,4 & & & 8 & 5,2 \\
\hline No C. total & 182 & 100 & 189 & 100 & 158 & 100 & 153 & 100 \\
\hline
\end{tabular}

Valores promedios de tres perfiles

No C = Número de cuadrículas impactadas del total observado

$\%$ = Porcentaje del total de cuadrículas impactadas

Número total de cuadrículas observadas $=800$

Las observaciones se realizaron en el momento de la floración

Los cultivos intercosechas o asociaciones se incorporaron a los 85 días.

\section{Impacto de las prácticas establecidas sobre los ren- dimientos de maíz y frijol.}

Los rendimientos obtenidos (Figura 2), muestran un discreto incremento en ambos segmentos, siendo mayores en el segmento con barreras de Gliricidia sepium y asociaciones de maíz + Stizolobium deeringianum, (400 y 170 kg/ha de maíz y frijol en granos respectivamente). Esta respuesta está en consonancia con la mejora de la fertilidad general, expresadas en el incremento de la porosidad estructural, la permeabilidad, aumento de la humedad residual en la sección receptora donde se acumulan los sedimentos, mayor abastecimiento de nutrientes por la misma causa y mayor desarrollo de las plantas que ocupan esos dos a tres metros próximos a la barrera en la sección receptora.

\section{LITERATURA CITADA}

ALFONSO, C.A.; RIVEROL, M.; PORRAS, P.; CABRERA,E.; LLANES, J. M.; HERNANDEZ, J.M. y SOMOZA, V. 1997. Las asociaciones maíz - leguminosas su efecto en la conservación de la fertilidad de los suelos.Agronomía Mesoamericana (C.R.) Vol. 8 (1) 65-73.

ALFONSO, C.A. 1987. Etude de la degradation des sols cultives en tabac, Cuba, province de Pinar del Río. Thése Docteur Ingenieur, INA-P.G. France. 187 p.

ALTIERI,. M. 1995. Una alternativa dentro del sistema. CERES. (Italia) 27 (4): $15-23$.

COLLINET, J.; MAZARIEGO, M. 1996. Rehabilitación de los suelos Volcánicos degradados utilizando abonos orgánicos Cuenca del Río Las Cañas - El Salvador. XLII Reunión del PCCMCA. 18 - 22 de Marzo. 23p. 
Da VEIGA, M. ; Do PRADO, L. 1993. Manual para la instalación y conducción de experimentos de pérdidas de suelos. Proyecto Regional GCP/RLA/107/JPN FAO. 32p.

FRANCISCO, N. 1995. Estabilidad, grado de desarrollo y productividad de terrazas de muro vivo para la conservación y mejoramiento desuelos agrícolas de laderas. Tesis de Maestría en Ciencias. Colegio de Posgraduados. Montecillo, México. 129 p.

GARCIA, A.M.; TRETO, E.; ALVAREZ, M. 1996. Estudio de la factibilidad agronómica y económica del empleo de los abonos verdes en la Agricultura. Resumen y Seminario Científico INCA. Cuba. Octubre de 1996.

HENRIQUEZ, G.R.; PROPHETE, E.; ORELLANA, C.L., 1992. Manejo Agronómico del Cultivo del Frijol, (Phaseolus vulgaris L.). De. CIAT-PROFRIJOL. 120 p.

MANICHON, H. 1982a. Influence des systemes de culture sur le profil cultural: élaboration d'une méthode de diagnostic basée sur l'observation morphologique. These Docteur Ingénieur, INA-PG, 214 p.
MARIN, D., 1996. Comparación ecofisiológica de los cultivares Tovar y Yaracuy de Canavalia ensiformis (L). , sembrados en dos localidades. I. Análisis de crecimiento. Agronomía Tropical. (Venezuela). 46 (1): 5 - 30.

MONNIER, G.; STENGEL, P.; FIES, J.C. 1973. Une méthode de mesure de densité apparente de petits agglomérats terreux. Aplication a l'analyse des systemes de porosité su sol. Annales Agronomiques. (France). 24 (5), 533-545.

TURRENT, F.A.; URIBE, G.; FRANCISCO, N.; CAMACHO, R. 1995 b. La terraza de muro vivo, para laderas del trópico subhúmedo de México II. Cambio en algunas propiedades físicas y químicas del suelo. Terra (México) 13(3): 299-316. SMCS.

URIBE, G.S. ; FRANCISCO, N. 1993. Manejo agronómico sustentable para laderas abruptas de Los Tuxtlas, Veracruz. Informe técnico. SARH- INIFAP-CIRGOC-CEPAP. Isla Veracruz. 